\title{
PKS 0537-286, the high red-shift FSRQ, giving new insights into the jet physics
}

\section{Eugenio Bottacini ${ }^{1}$, Jochen Greiner}

Max-Planck-Institut fuer extraterrestrische Physik

Giessenbachstrasse, 85748 Garching, Germany

E-mail: eub@mpe.mpg.de, jcg@mpe.mpg.de

\section{Marco Ajello}

Stanford Linear Accelerator Center/KIPAC

2572 Sand Hill Road, Menlo Park, CA 94025

E-mail: majelloeslac.stanford. edu

\section{Arne Rau}

California Institute of Technology

1200 East California Blvd, Pasadena, CA 91125

E-mail: arnedastro.caltech.edu

\section{Elena Pian}

Istituto Nazionale di Astrofisica

Via G. B. Tiepolo, 11, 34143 Trieste, Italy

E-mail: piandoats.inaf.it

\section{Aybüke Küpcü Yoldaş}

European Southern Observatory

Karl-Schwarzschild-Strasse 2, 85748 Garching, Germany

E-mail: ayoldaseso.org

\section{Eliana Palazzi}

Istituto Nazionale di Astrofisica

Via Gobetti 101, 40129 Bologna, Italy

E-mail: palazziliasfbo.inaf.it

The 7th INTEGRAL Workshop

Copenhagen, Denmark

September 8- 112008

\footnotetext{
$1 \quad$ Speaker
} 
We have performed a multiwavelength campaign on the high red-shift FSRQ PKS 0537-286 during October - November 2006 and February 2008. The automatic optical and near-infrared telescope (REM) and the Gamma-Ray Burst Optical and Near Infrared Detector (GROND) observed the source simultaneously to X-ray and hard X-ray satellite pointings with INTEGRAL/ISGRI, Swift/XRT and RXTE that allowed covering a wide range of energies. At $\mathrm{X}$-ray energies no significant variability was found. The Swift/BAT light curve over two years of survey showed a constant flux level. We find a rather flat high energy spectrum and absorption at soft X-rays in excess to the Galactic value, as previously found in other FSRQ [10][13]. We propose that these features are the signature of bulk Comptonization of soft X-ray photons.

\section{Introduction}

The spectral energy distribution (SED) of blazars is dominated by non-thermal continuum. The relativistic jets producing the typical two hump structure from radio to gamma-rays and sometimes to $\mathrm{TeV}$ band [11] extend over kpc scale. According to whether jets are observed from the side or pole-on, they give rise to radio galaxies or blazar objects [21]. Within this framework multiwavelength studies of blazars give a unique insight into the jet physics due to emission enhancement through relativistic beaming. These observations over several decades of energy started to be carried out more than ten years ago and lead to different emission models. But still the question, on how the central super massive black hole (SMBH) transfers its gravitational energy to the relativistic moving plasma, is asking to be solved.

The overall blazar emission is successfully interpreted in the view of the synchrotron and inverse-Compton models. The first peak at lower energies is dominated by synchrotron radiation, while at higher energies the second component is due to inverse-Compton scattering. The prevailing picture of the emission mechanism foresees the emitting plasma to be accelerated within the jet. Cooling and acceleration of these relativistic particles leads to variability in the measured spectrum caused by shocks within the jet. In this scenario also the external components [9] to the jet, like the accretion disk surrounding the central SMBH, play a fundamental role, injecting external seed photons scattered by the broad line region (BLR) into the jet.

The importance of high red-shift blazars is related to the evolution studies of SMBH in the early Universe. Indeed, their accretion rate is related to the circumnuclear environment that should be revealed in terms of spectral emission features in the X-ray energy band. Their high energy spectral properties make them suitable candidates for the recently launched FERMI (formerly GLAST) gamma-ray mission and thus ideal targets for further probe of gamma-ray horizon [19].

The X-ray spectra of high red-shift blazars are generally hard and exhibit occasionally absorption in the soft energy range in excess to that due to the Galactic hydrogen column density [22] [24]. 


\section{The observation campaign}

\subsection{The source}

PKS 0537-286, at $\mathrm{z}=3.104$ [23], is one of the most luminous known high red-shift quasar. First detected at radio frequencies [5], it was observed at X-rays by the Einstein observatory [25] and then studied with ASCA [7] [17], ROSAT [10], XMM [14] and Swift [15]. These observations showed an extremely luminous quasar $\left(\mathrm{L}_{\mathrm{x}}=10^{47} \mathrm{erg} \mathrm{s}^{-1}\right.$ in the $0.1-2 \mathrm{keV}$ range $)$. A further characteristic is its particularly hard spectrum and a weak iron $\mathrm{K} \alpha$ emission and reflection features. Moreover Sowards-Emmerd et al. identified PKS 0537-286 as probable counterpart of the EGRET source 3EG J0531-2940 [18].

\subsection{Observations}

The source was proposed to be monitored by INTEGRAL. Due to the fact that the prior Swift/BAT light curve over 9 months of exposure [1] was consistent with a constant flux level, the INTEGRAL pointings were proposed in non-contiguous time intervals gaining an exposure of 1 Ms.The obervations were performed during revolutions: $0493,0494,0549,0550,0552$, 0558. These observations were performed with a rectangular dithering pattern. By instrument design, only few dither pointings produced a useful JEM-X spectrum of the source being offaxis for most of the time. The source is only marginally detected with IBIS/ISGRI [12] in the 17 - $55 \mathrm{keV}$ band. Using the detected count rate, we performed data cleaning. Most recent matrices available for standard software were used for spectral analysis. We used 296 science windows for a total amount of $981 \mathrm{ksec}$ of exposure time on the source. The source is detected with ISGRI at a significance level of 5.6 sigma. Data screening was performed according to the median count rate with respect to each science window and their distribution. Due to the low detection level a spectrum cannot be fitted.

The Burst-Alert Telescope (BAT, [3]) on board the Swift satellite is a coded-mask telescope operating in the $15-200 \mathrm{keV}$ energy range. We selected all observations comprised in the time span January 2005 - March 2007. The data were processed using HEASOFT 6.3 and following the recipes presented in [2]. The spectrum and the light curve of PKS 0537-286 were extracted using the method presented in [1].

RXTE All Sky monitor observed PKS 0537-286 for 9.9 ksec, starting from November 1st 2006. We reduced the data using the standard reduction script REX included in the HEAsoft6.0.4 package.

The X-Ray Telescope [6] on board the Swift observed the blazar on October 27th, 30th, 31st 2006 and February 10th, 12th 2008. The monitoring campaign was carried out within five observations. Data processing, screening and filtering were done using the FTOOL xrtpipeline comprised in the HEASOFT 6.3 distribution.

Simultaneously to the RXTE and Swift/XRT pointings in 2006, photometry with REM was performed in 5 optical energy bands (V, R, J, I, H).

GROND observed the source simultaneously to the Swift/XRT pointings in 2007. 


\section{X-ray and hard X-ray spectra}

For the Swift/XRT spectra, a power law with a fixed galactic absorption $\left(2.0 \times 10^{20} \mathrm{~cm}^{-2}\right)$ yielded an unacceptable fit. Leaving the absorption value to be fitted freely a substantial improvement of the reduced chi-square (0.9) was obtained for each spectrum. The lower limit of the $\mathrm{N}_{\mathrm{h}}$-value is $5.3 \times 10^{20} \mathrm{~cm}^{-2}$, that is well above the galactic value. While the spectral index assumes values around 1.5. The absorption parameter is in good agreement within the five observations and so does the spectral index.

It is unlikely that an excess column density is in origin galactic. It would require a factor $\sim 3.5$ of increase in the galactic column density toward the direction of the source. Also the intergalactic origin is highly improbable because the line of sight should intercept several systems having very high column density. The intrinsic absorber hypothesis is much debated. The best evidences for warm absorbers, like $\mathrm{O}$ absorption edge and $\mathrm{Fe} \mathrm{M}$-shell, are down shifted outside our observed range $(0.3-10 \mathrm{keV})$, being in the rest frame's energy band within $0.5-0.9 \mathrm{keV}$.

None of the X-ray and hard X-ray observations showed flux variability. We therefore fitted all the spectra simultaneously. Figure 2 (left panel) shows the joint spectrum of the instruments Swift/XRT, RXTE and Swift/BAT obtained during the multiwavelength campaign.

A theoretical interpretation to the excess absorption and hard spectral index in the X-ray spectra is given by [8] who foresee a peaked seed photon energy distribution. The seed photons, arising from the innermost part of the accretion disk and entering the jet through the reprocessing in the BLR, are bulk comptonized in the jet. Thus the entire spectrum is the contribution of relativistic Compton and bulk Compton, which can be approximated by the sum of a power law and black body components.

Bridging the energy gap between Swift/XRT- and Swift/BAT-data with RXTE, our data are very suitable for the determination of the above mentioned spectral features. Therefore we fitted in addition to a power law also a black body component gaining an equal good fit result (reduced chi-square 0.8 for 142 degrees of freedom) as previously but with better residuals. The f-test probability of $97.2 \%$ confirms the additional parameter. Figure 1 shows the comparison between residuals of the fit of power law + black body and simple absorbed power law. The resulting seed photon temperature of $8.6 \mathrm{keV}$ is in good agreement with the predicted one by the authors of the earlier mentioned paper. This high value for the temperature of the seed photons is not surprising since in the observer frame it is blueshifted by the bulk Lorentz-factor and the Doppler-factor after the scattering.

A further model describing bulk Comptonization motion is given by [20] whose model is self-consistent bulk motion Comptonization rather than the sum of two components (as used previously). Again we obtained a good fit with reduced chi-square of 0.9 (with 141 degrees of freedom) by fitting the BMC-model. The seed photon temperature of $0.2 \mathrm{keV}$ is in good agreement with the one expected, considering a geometrically thin and optically thick accretion disc, having surface temperature (multi-color black body) profile as in [16]. 


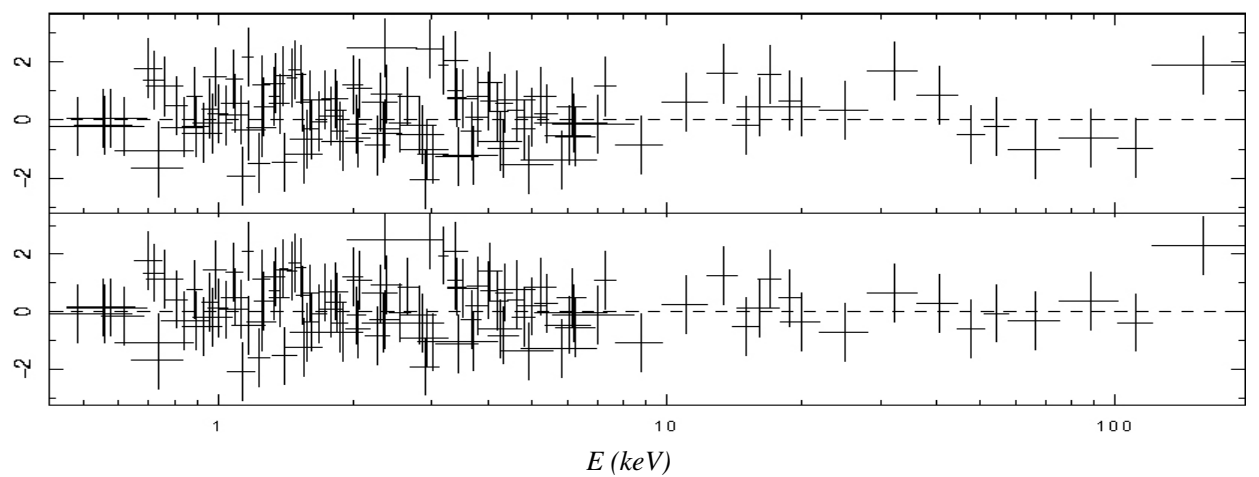

Figure 1: The comparison between the residuals obtained with a power law + black body model (lower panel) and a simple absorbed power law model (upper panel) is shown. It is apparent that in the latter case the data points at energies larger than $50 \mathrm{keV}$ are better fitted.

The confidence contours of fitted seed photon temperature and alpha-parameter of the fitted BMC model are shown at confidence levels of 68\%, 90\% and 99\% (see figure 2, right panel).
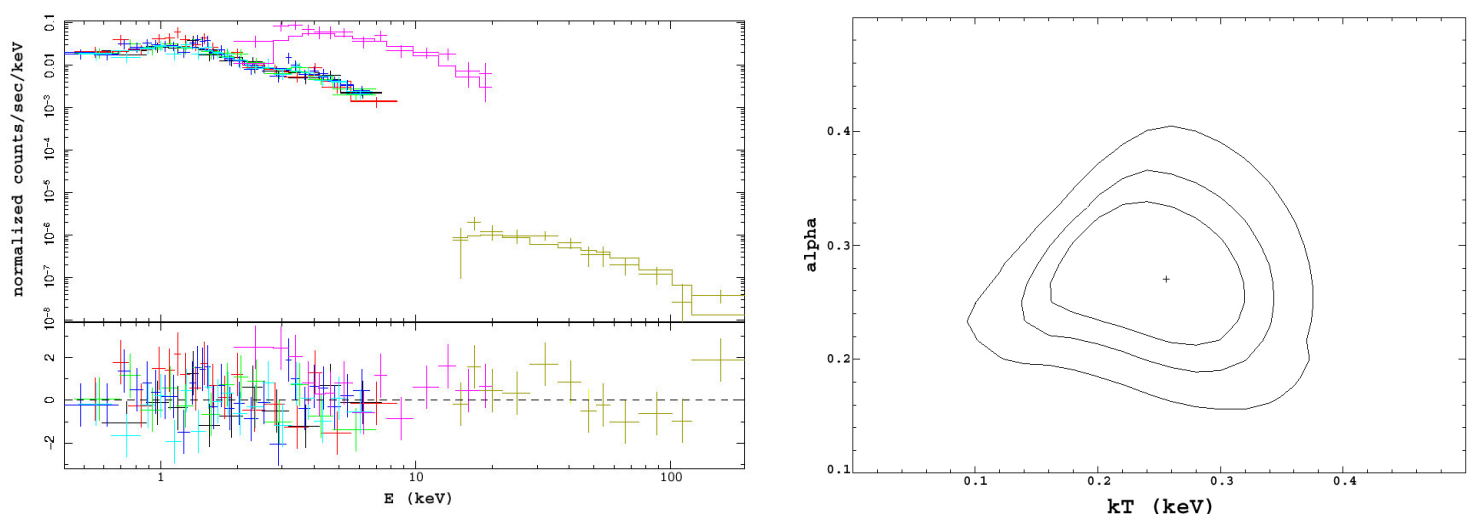

Figure 2: Left panel shows the joint spectrum of the instruments Swift/XRT, RXTE and Swift/BAT obtained during the multiwavelength campaign. The fit to the data points is an absorbed powerlaw of spectral index 1.3. Right panel shows the confidence level contours at $68 \%, 90 \%$ and $99 \%$ of the parameter kT (indicating the temperature of the seed photons) and alpha (indicating the efficiency of the Comptonization) of the BMC model [20].

\section{Conclusions}

We have conducted a multifrequency campaign on PKS 0537-286 on five different epochs. At X-rays the source showed no flux variability. We probe the spectral flattening for the source and show that it is physically associated to it. We detect spectral X-ray features, related to bulk Comptonization scattering, predicted by [8]. Furthermore, the spectra can be fitted with the bulk motion comptonization model (BMC) [20] gaining a reduced chi-square of 0.9. Thus, we find the source's jet to be matter dominated. This finding can have an impact on cosmological evolution of blazars. Indeed, Boettcher and Dermer (2002) proposed a scenario 
where BL Lacs are the final stage of FSRQ [4]. As the accretion power drops with time, less gas and dust are left. Thus, FSRQs at high red-shift are expected to be matter dominated.

\section{References}

[1] M. Ajello et al., 2008, ApJ, 673, 96

[2] M. Ajello et al., 2008b, ApJ, 678, 102

[3] S. D. Barthelmy et al., 2005, SSRv, 120, 143

[4] M.Boettcher, C.D. Dermer, 2002, ApJ, 564, 86

[5] J. G. Bolton et al., 1975, Aus. J. Phys. Ap. Suppl. 34, 1

[6] D. N. Burrows et al., 2005, SSRv., 120, 165

[7] M. Cappi et al., 1997, ApJ, 478, 492

[8] A. Celotti et al., 2007, MNRAS, 375, 417

[9] C.D. Dermer et al., 1992, A\&A, 256, 27

[10] F. Fiore et al., 1998, ApJ, 492, 79

[11] G. Fossati et al., 1998, MNRAS, 299, 433

[12] F. Lebrun et al., 2003, A\&A, 411, 14

[13] J.N. Reeves \& M.J.L. Turner, 2000, MNRAS, 316, 234

[14] J.N. Reeves et al., 2001, A\&A, 365, 116

[15] R.M. Sambruna et al., 2007, ApJ, 669, 884

[16] N.I. Shakura \& R.A. Sunyaev, 1973, A\&A, 24, 337

[17] J. Siebert et al., 1996, A\&A, 307, 8

[18] D. Sowards-Emmerdet al., 2004, ApJ, 609, 564

[19] F. W. Stecker et al., 2006, ApJ, 648, 774

[20] L. Titarchuk et al., 1997, ApJ, 487, 834

[21] C.M. Urry \& P. Padovani, 1995, PASP, 107, 803

[22] M.A. Worsley et al., 2004, MNRAS, 350, 67

[23] A.E. Wright et al., 1978, 226, 61

[24] W. Yuan et al., 2005, MNRAS, 358, 4

[25] G. Zamorani, et al., 1981, ApJ, 245, 357 